\title{
EDITORIAL \\ Impact of COVID-19 on neurosurgery resident research training
}

\author{
Victoria E. Clark, MD, PhD \\ Department of Neurosurgery, Massachusetts General Hospital, Boston, Massachusetts
}

$\mathrm{T}$ He light flashed red on the card reader outside the main entrance of the research institute. I waited for a moment, still holding my ID badge as if another attempt would yield a different result. It was official: I was locked out of the lab. My experiments would be paused indefinitely until Boston, and the rest of the country, gradually returns to a new normal as a result of the COVID-19 pandemic. Fortunately, the institute had received several days of advanced notice to ramp down in-person experiments. My genetically modified cells, which I had been tending like orchids for months and which I hoped would mirror critical features of meningioma biology, were frozen in liquid nitrogen. My scientific training was in a similar state of indefinite suspended animation. I pictured this scene repeating itself thousands of times throughout the country as the pace of biological research unceremoniously ground to a halt.

Even without a global pandemic, there are multiple challenges to scientific productivity during the neurosurgery residency research years. While we learned to dissect tumor planes and to instrument the spine as junior residents, science continued to advance at warp speed. Picking up the pipette that you put down half a decade ago requires extensive planning, organization, and a committed mentor. In practice, it feels like parachuting onto a moving train. In contrast to our $\mathrm{PhD}$ postdoctoral colleagues, the block of time we dedicate to research is predetermined and fixed, imbuing project feasibility with utmost importance. Most neurosurgery residents continue to have some clinical duties during the research years, which objectively slows research progress but provides exceedingly valuable practice for learning to balance a career combining neurosurgery and science. On the subject of balance, many residents have children during the research years due to multiple logistic reasons, including to minimize the impact on the neurosurgery clinical service.

The spread of COVID-19 has intensified these obstacles to our scientific training. Despite best efforts at advanced experimental planning, few residents could have reasonably accounted for an indefinite block of time in which we would be unable to physically conduct lab benchwork. The stop clock continues running on our research years and requesting to extend this research time would increase the clinical burden on our coresidents and negatively impact our personal surgical case volume. Scientific conferences, with their rich opportunities for networking, have been cancelled. Research residents are gladly taking on additional shifts in the hospital to cover for our ill neurosurgical colleagues and anticipate a likely need to be mobilized to assist the general medicine services in the direct care of COVID-19 patients. For many residents, this increase in clinical responsibilities exactly coincides with a dramatic decrease in childcare options, as numerous daycares and schools face prolonged closures.

One approach for residents to use this time efficiently is to scale up research activities that can be performed remotely. For example, we can continue to generate new scientific knowledge by shifting toward bioinformaticsbased projects or performing chart-based/clinical analyses. We can focus on our scientific writing, including synthesizing existing data, penning topic reviews or book chapters, and applying for grants. The wide availability of online tools such as video conferencing and workflow software can be employed to plan the most efficient use of time and resources once lab members are allowed back in the building. However, while we can and should divert our efforts into salvaging productivity through remote work, the ban on wet bench research remains devastating for many projects. It is conceivable that the negative impact on our scientific productivity will ultimately limit our career prospects.

By refraining from laboratory-based research, we are doing our part to "flatten the curve." However, the viral pandemic that has essentially shut down wet bench re- 
search has paradoxically accentuated its necessity and highlighted the critical need for the continued training of physician-scientists. On a societal level, I absolutely agree with the efforts to minimize the devastating impact that unchecked SARS-CoV-2 infections will have on the medically vulnerable and our healthcare system at large. The sheer magnitude of the projection that hundreds of thousands of people could die in the United States of COVID-19 is difficult to fully comprehend. But for many of our individual patients, their underlying neurosurgical disease remains the highest threat to their survival. Even in a time of pandemic, aneurysm walls are stretching thin, undetected high-grade gliomas are silently blooming, and fontanelles are just starting to bulge. The acuity and importance of neurosurgical research endures, and it cannot all be performed remotely.

As neurosurgeon-scientists, we must advocate for safe, socially distant methods to continue wet bench research on neurosurgical disease during the COVID-19 pandemic. Potential solutions include working in designated shifts so that no two researchers are in the same room simultaneously, only performing the minimal essential in-person experiments, and parallelizing projects through increased collaboration. Shared resources, including microscopes and fume hood sashes, must be cleaned with antiviral agents between users. Pending resolution of nationwide supply concerns for hospital workers, masks can be considered to prevent viral spread between researchers. As antibody-based serological tests become clinically available, documented immunity to COVID-19 could be used to identify researchers who can safely return to work.

If doctors are frontline soldiers in the war on human disease, biomedical researchers are the bullet makers. Our patients deserve a full armamentarium. The path to accelerate the end of the COVID-19 pandemic and to propel neurosurgical care forward is to find a safe way to foster rapid scientific progress. Locking the doors to our nation's research institutes is not the answer.

https://thejns.org/doi/abs/10.3171/2020.4.JNS201034

\section{Disclosures}

The author reports no conflict of interest.

\section{Correspondence}

Victoria E. Clark: victoria.clark@mgh.harvard.edu.

INCLUDE WHEN CITING

Published online April 24, 2020; DOI: 10.3171/2020.4.JNS201034. 